\title{
A Study on the Impact of Nodes Density on the Energy Consumption of LoRa
}

\author{
https://doi.org/10.3991/ijim.v15i14.19825
}

\author{
Aizat Faiz Ramli ( $\left.{ }^{\varpi}\right)$, Muhammad Ikram Shabry, Mohd Azlan Abu, \\ Hafiz Basarudin \\ Universiti Kuala Lumpur British Malaysian Institute, Kuala Lumpur, Malaysia \\ aizatfaiz@unikl.edu .my
}

\begin{abstract}
LoRaWAN is one of the leading low power wide area network (LPWAN) technologies that compete for the formation of big scale Internet of Things (IoT). It uses LoRa (Long Range) protocol to achieve long range, low bit rate and low power communication. Large scale LoRaWAN based IoT deployments can consist of battery powered sensor nodes. Therefore, the energy consumption and efficiency of these nodes are crucial factors that can influence the lifetime of the network. However, there is no coherent experimental based research which identifies the factors that influence the LoRa energy efficiency at various nodes density. In this paper, results on measuring the packet delivery ratio, packet loss, data rate and energy consumption ratio (ECR) to gauge the energy efficiency of LoRa devices at various nodes density are presented. It is shown that the ECR of LoRa is inversely proportional to the node's density and that the ECR of the network is smaller at higher traffic indicating better network energy efficiency. It is also demonstrated that at high node density, spreading factor SF of 7 and 9 can improve the energy efficiency of the network by 5 and 3 times, respectively, compare to SF 11 .
\end{abstract}

Keywords-IoT, LoRa, LoRaWAN, energy efficiency, node density

\section{Introduction}

The demand of IoT devices is expected to grow exponentially in the next decade fuelled by the industry sector [1]-[3]. IoT offers opportunity to revolutionize the manufacturing systems and operation [4]-[6]. However, the limited wireless channel or frequency dimension means that concurrent access to the channels by these devices will be difficult to achieve and thus increases delay and stifle the network performance.

There are various IoT protocols with varying degree of performance in terms of latency, coverage, range and energy consumption. One such protocol is LoRaWAN (Low Power WAN Protocol for the Internet of Things) which uses LoRa to interact with gateways through a single hop. LoRaWAN is one of the leading LPWAN technologies that compete for the formation of big scale IoT. LoRaWAN is a data-link layer with long range, low bit rate and low power. It is expected to provide a solution 
for the connection of billions of devices in the future [7]. IoT applications are typically battery powered and/or energy constraint devices that performs complex computational process. Therefore, optimizing the energy efficiency of LoRaWAN and LoRa are a major consideration for any IoT networks.

There have been various research on studying and modelling the energy consumption of LoRa and LoRaWAN [8]-[11], these research assumed a constant nodes density thus may not be applicable to a large scale and high nodes density LoRaWAN networks. The purpose of this paper is to measure the energy consumption ratio ECR of LoRa supporting various nodes density. The measurement will be used to study the factors that can affect the energy efficiency of high density LoRaWAN networks. This paper is organized as follows. Section 2, provides discussion on the findings of related research. Section 3, describes the methodology and experimental set-up to measure LoRa energy efficiency in various scenarios. The measurement results are presented and discussed in section 4. Finally, conclusions are drawn.

\section{Review on LoRa and LoRaWAN Energy Consumption}

\subsection{LoRa and LoRaWAN protocol}

The architecture or the topology of LoRaWAN is considered as "star of star". While the physical layer (LoRa) enable the long-range link, the communication protocol and network system architecture are defined. The protocol influences and determine the lifetime battery of a node, the network capacity, security, quality of service (QoS) and also the number of applications served by the network [7].

For a LoRa network infrastructure to manage the individual data rates and maximize the lifetime of the battery of each device via radio frequency (RF) output, the adaptive data rate (ADR) scheme is used. The end devices are not associated with a specific gateway in the traditional cellular network. For instance, the packet received from the end devices are routed after the information about the reception quality (QoS) is added. So, an appropriate gateway needs to be chosen by the end device. The gateway must be logically transparent to the end-devices so that a response (if any) can be sent [7].

LoRaWAN devices can access the communication channel using pure ALOHA medium access control (MAC) protocol [8],[9]. In ALOHA, packet generated by devices can be directly transmitted to the communication channel without having the need to first perform carrier or collision detection. The simplicity of this protocol reduces the communication overhead, cost and crucially it minimizes the power consumption of transmitting devices [10]-[12]. Depending on application QoS requirements, end devices of LoRaWAN can be configured to operate in three class types: class A, B and C. Table 1 provides summary on the technologies and expected quality of service QoS of LoRaWAN. 
Table 1. LoRaWAN key parameters

\begin{tabular}{|l|l|}
\hline \multicolumn{1}{|c|}{ Parameters } & \multicolumn{1}{c|}{ Values } \\
\hline Frequency Band & Unlicensed ISM bands \\
\hline Modulation & SS Chirp (chirp spread spectrum) \\
\hline Range & $10 \mathrm{~km}$ (urban) 50km (rural) \\
\hline Topology & Star on Star \\
\hline Uplink data rate & $300 \mathrm{bps}-50 \mathrm{kps}$ \\
\hline Security & Advanced Encryption Standard (AES-128) \\
\hline
\end{tabular}

\subsection{Related works}

Kurtoglu et al. [13] compares the energy consumption used in a special case of wireless sensor network which is installed continuously (linearly) over a long distance for the purpose of monitoring. For instance, pipelines and transmission line. From the energy consumption model that was developed (based on appropriate network architecture for linear wireless sensor network), the energy consumption of LoRaWAN and ZigBee were compared. The network architecture model consists of five cluster nodes $(\mathrm{CN})$ in a cluster and five clusters in a supercluster. It is found through their model that the clusters in LoRaWAN networks consumed $5.3642 \mathrm{~J}$, which significantly less energy than Zigbee which consumed 20.3731 J.

Cheong et al.[14] conducted experimental set up to analyze the energy consumed in LoRa devices operating in 3 different classes (A, B and C). Current levels of the devices were measured in four different operation modes; transmit, receive, idle and sleep operating at spreading factor 7 and 11. It is found that in their experimental, the current levels for different operation mode does not show any clear trend and display the same order of magnitude over payload size and spreading factor. Using higher spreading factor will also higher energy consumption particularly which will shorten the battery lifespan. For class A, the packet interval can significantly affect the total device power consumption.

An energy consumption model for sensors using LoRa and LoRaWAN technologies were developed in [15] The model is based on LoRaWAN class A. It is shown that LoRa parameters such as spreading factor SF, packet interval and coding rate can affect the energy consumption. Other important factor that can influence the lifetime of a sensor is the operating frequency of micro-controller. Higher operating frequency will reduce the battery lifetime of sensor and LoRa.

\section{$3 \quad$ Methodology and Experimental Setup}

An Arduino Uno board with LoRa-RFM Shield were used as transmitter and receiver. The power consumption of the device in different modes (sleep mode, transmitting, receiving and idle mode) were measured in terms of power, voltage, current, resistance and power. Fig. 1 shows the block diagram of LoRa transmitter and receiver nodes used as the foundation of the research. 


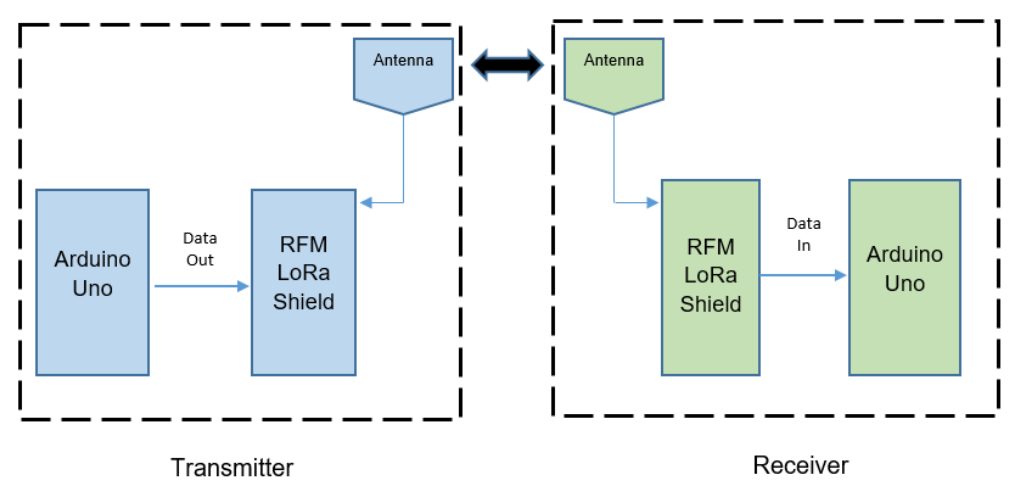

Fig. 1. Block Diagram for Transmitter \& Receiver

Fig.2 shows the star topology used to measure the transmission of the network supporting different nodes density in terms of its energy efficiency. The numbers of routers will be varied from 1 to 5 .

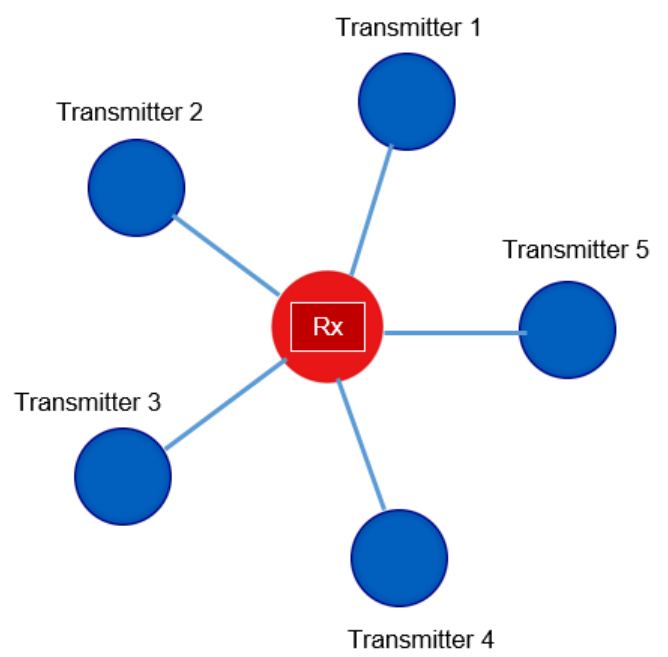

Fig. 2. Star topology to measure transmission of network with 5 nodes

\subsection{Performance metrics}

The followings are the performance metrics used to analyze the throughput and QoS of LoRa networks supporting various nodes density at different SF values [16],[17]. The energy consumption ratio (ECR) metrics will be used to assess if the performance benefits (in terms of throughput) obtained by increasing node density outweigh the additional energy consumed by the networks [19]. 
- Power consumption: The power consumption of LoRa is measured using a Log4 data logger [17] The LoRa power consumption was measured in transmit, receive, sleep and idle mode.

- Packet Delivery Ratio (PDR): PDR provides indication on efficiency of a network to successfully receive packets. It is defined as the ratio of received packets to the number of packets transmitted as shown in (1).

$$
\text { PDR }(\%)=\frac{\text { Total Packet Received }}{\text { Total Packet } \text { Trasnmitted }} \times 100
$$

- Packet Loss: Packet Loss is the unsuccessfully transmitted packet to arrive at their destination (receiver). Packet Loss is calculated (in percentage) based on the ratio of the unsuccessful packet to the total number of the transmitted packet as shown in (2).

Packet Loss $(\%)=\frac{\text { Total Packet Transmitted }- \text { Total Packet Received }}{\text { Total Packet Trasnmitted }} \times 100$

- Data Rate: The speed of data transmission from a transmitter to receiver is called data rate. It is measured in bytes per second. The data rate in bytes per second of the network is calculated using (3).

$$
\text { Data Rate }(\text { bytes } / \text { second })=\frac{\text { Total Data Received }}{\text { Time }(\text { seconds })}
$$

- Energy Consumption Ratio (ECR)": ECR is defined as the energy consumed to transmit one byte of data [19]. It is the ratio of the total energy consumed by LoRa to the total data received. ECR of the network was obtained in Joule per bytes as given in (4).

$$
\text { ECR }(J / \text { bytes })=\frac{\text { Total Enegy }}{\text { Total Data Received }}
$$

\subsection{Experimental setup}

The experimental set up to measure the aforementioned parameters for varying the nodes density was achieved by gradually increasing the number of transmitter from 1 to 5 as shown in Fig 3. All the transmitter nodes will continuously transmit 10 bytes of packet and set to the same settings. The parameter setting of LoRa devices is summarized in Table 2.

Table 2. LoRa parameters setting

\begin{tabular}{|l|l|}
\hline \multicolumn{1}{|c|}{ Parameters } & \multicolumn{1}{c|}{ Values } \\
\hline Frequency Band & $915 \mathrm{MHz}$ frequency band \\
\hline Transmit Power & $13 \mathrm{~dB}$ \\
\hline Coding rate & $4 / 5$ \\
\hline Bandwidth & $125 \mathrm{kHz}$ \\
\hline Packet size & 10 bytes \\
\hline Spreading Factor SF & $7,9,11$ \\
\hline
\end{tabular}


To analyse the energy efficiency of LoRa network at different traffic load, packet time interval was set at 1,2 and 3 seconds. The experiment was also repeated at spreading factor SF of 7, 9 and 11 . The measurement were conducted for a total of 10 minute with 1500 samples at certain number of transmitter with specific setting of packet time interval and SF. The data is recorded after 10 seconds ensure that network has stabilized.

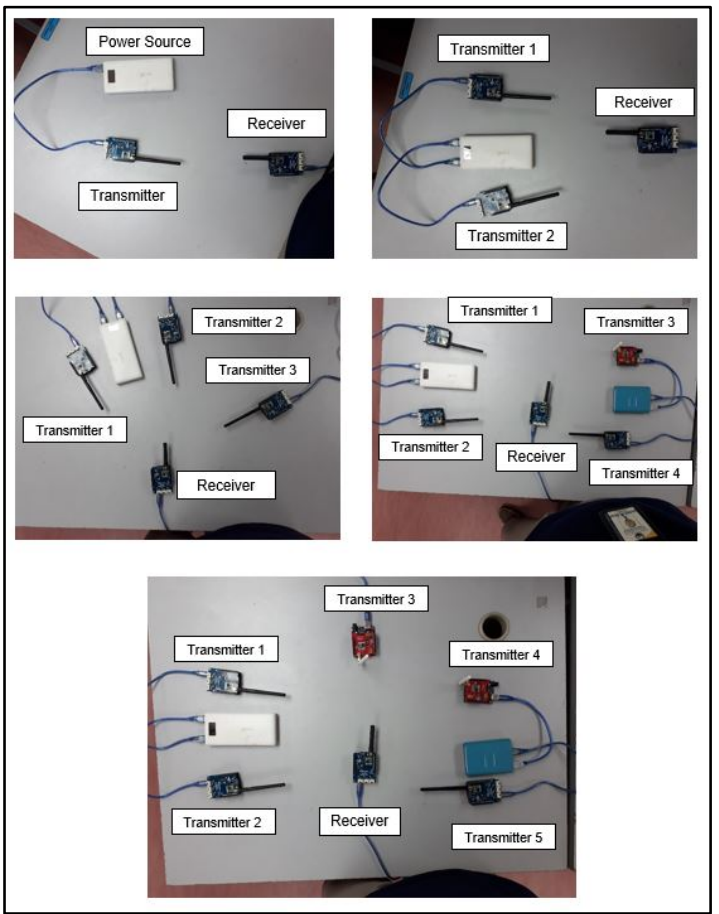

Fig. 3. Network with different node densities test practically

\section{$4 \quad$ Results and Discussion}

The result on the percentage of PDR at varying nodes density and packet interval time is shown in Fig 4. From the result, it can be seen that as the number of transmitter increases i.e. higher nodes density, the percentage of the PDR decreases. When only one transmitter is used, the percentage of PDR is almost $100 \%$ which means the receiver successfully received all the transmitted packets. The duration of the time interval between successive packets also influence the efficiency of the LoRaWAN network to successfully receive packet. A relatively low time packet interval duration causes more packet collision thereby reducing the network throughput. 


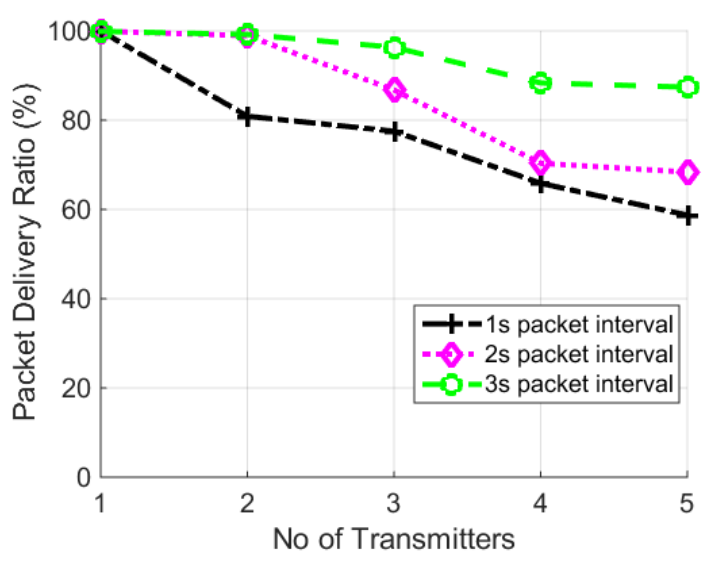

Fig. 4. PDR of LoRa at different nodes density

Packet loss is the number of unsuccessful packets unable to be successfully received by the receiver. As shown in Fig.5, the packet loss increases at higher nodes density due to higher probability of packet collision.

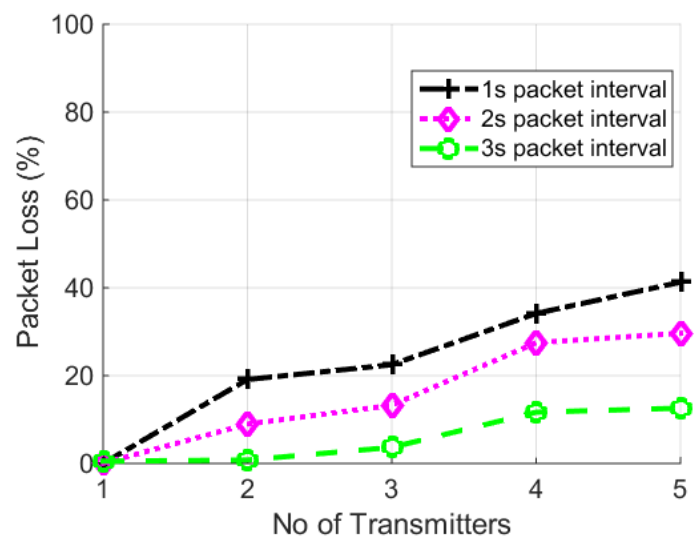

Fig. 5. Packet loss of LoRa at different nodes density

Fig. 6 and 7 illustrates the data rate of LoRa at different traffic load and SF respectively. It can be seen that at low traffic load (such as at 3 second packet interval), the data rate of LoRa is directly proportional to the number of transmitters. 


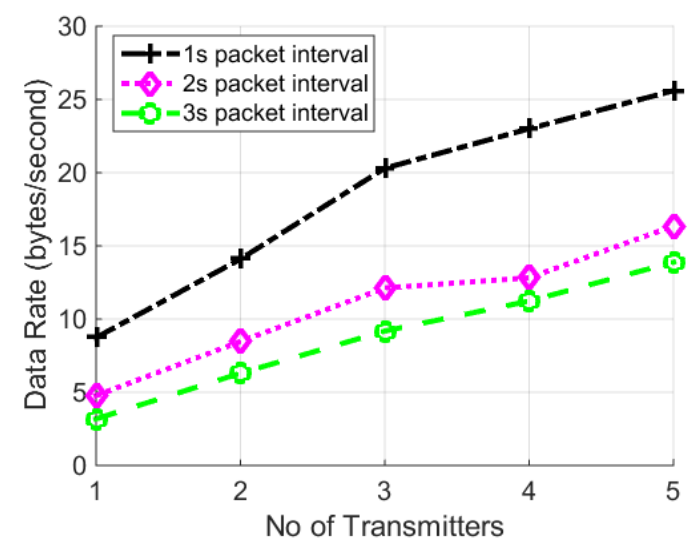

Fig. 6. Data rate performance of LoRa against nodes density for various traffic load

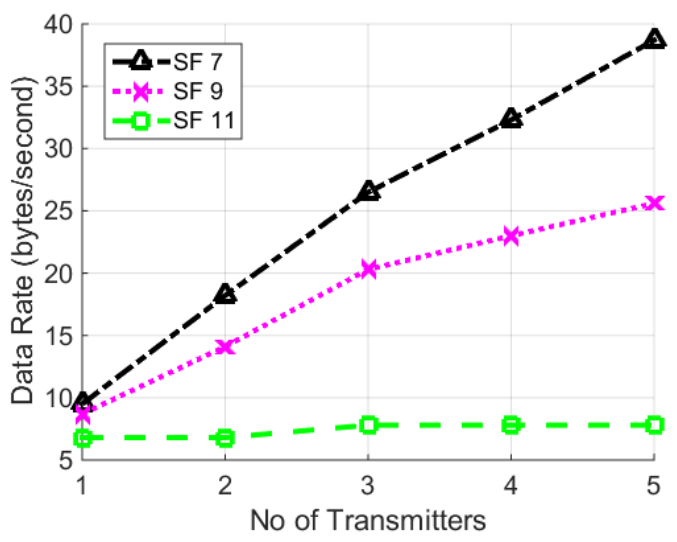

Fig. 7. Data rate performance of LoRa against nodes density operating at various SF

ECR metric is used in this research to quantify the energy efficiency of LoRa and LoRaWAN networks. It can be seen in Fig 8, that the ECR of LoRa is inversely proportional to the nodes density thus signify better energy efficiency when there are more nodes in the network. At high traffic load (small packet interval) the nodes in the network are almost continuously in transmission mode thereby consuming more power.However, the total data successfully received is also significantly higher and offset the additional power consumed thus results in smaller ECR and better efficiency 


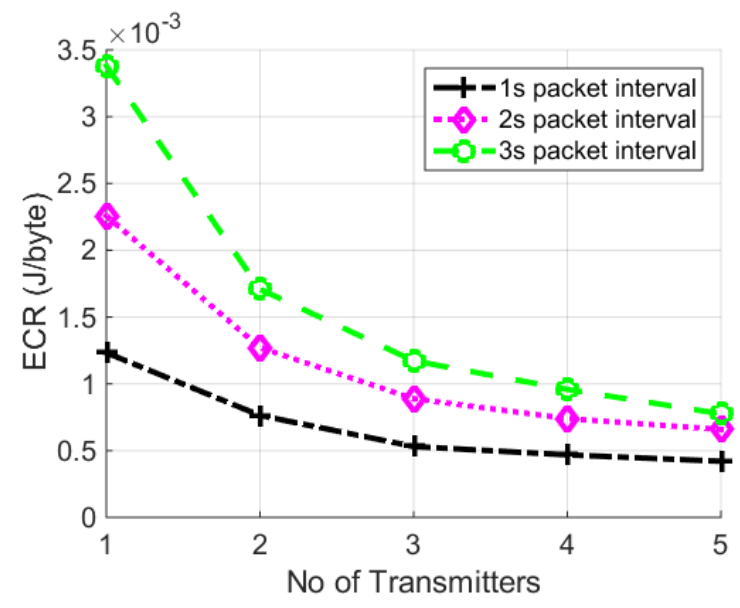

Fig. 8. Energy efficiency of LoRa against nodes density for various traffic load

Larger spreading factor SF will result in reducing LoRa communication data rate [20] as proven in the result shown in Fig.7. Larger SF will therefore increase the duration of packet transmission to time thereby consuming more power. Fig. 9 shows that at high node density, SF of 7 and 9 are approximately 5 and 3 times more energy efficient, respectively, compared to SF 11.

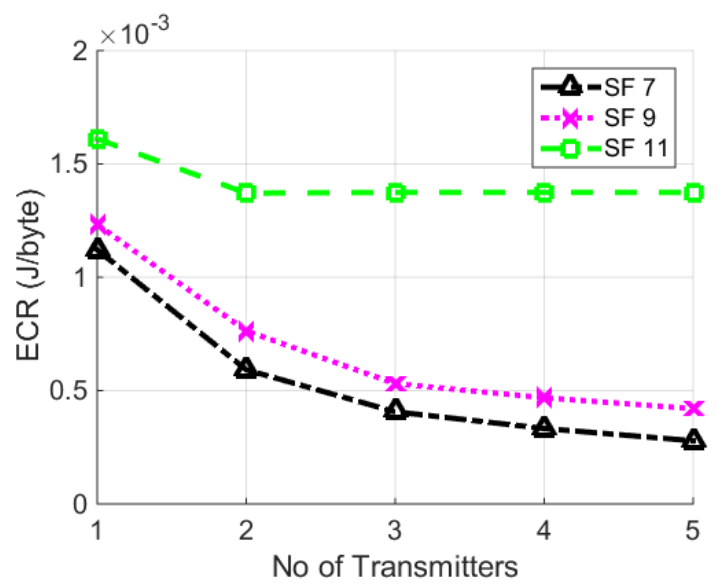

Fig. 9. Energy efficiency of LoRa against nodes density operating at various SF

\section{Conclusion}

In this research it is found that adding additional nodes in the network will improve the energy efficiency as measured in Joules per bytes for LoRa and LoRaWAN. In- 
creasing number of nodes results in significantly more data being generated and received which offsets the additional power consumed by the networks.

It is also found that the spreading factor SF has a significant impact on the energy efficiency of LoRa particularly at higher nodes density. It is recommended that a large-scale high density LoRaWAN deployment to consider the use of small SF values to obtain higher transmission data rate and reduce duration of nodes being in transmission mode thereby increasing the networks energy efficiency.

The limitation on the findings presented in this paper is the star topology and line of sight communication used to measure the energy efficiency of the network. Future direction of this research is to conduct experiment on LoRa energy efficiency using mesh topology in which all nodes can be both transmitter and receiver the sensor nodes will be able to communicate with each other via multi-hop links [21].

\section{Acknowledgement}

This research was supported by the Malaysian Ministry of Higher Education under Fundamental Research Grant Scheme (FRGS) (Grant number: FRGS/1/2019/TK04/UNIKL/02/22)

\section{$7 \quad$ References}

[1] Perera, C., Liu, C. H., Jayawardena, S., \& Chen, M. (2015). A Survey on Internet of Things from Industrial Market Perspective. IEEE Access, 2, 1660-1679. https://doi.org/10.1109/access.2015.2389854

[2] A. Kumar, M. Zhao, K. Wong, Y. L. Guan and P. H. J. Chong, "A Comprehensive Study of IoT and WSN MAC Protocols: Research Issues, Challenges and Opportunities," in IEEE Access, vol. 6, pp. 76228-76262, 2018. https://doi.org/10.1109/access.2018.2883391

[3] RAHMADYA, Budi; ZAINI, Zaini; MUHARAM, Mumuh. IoT: A Mobile Application and Multi-hop Communication in Wireless Sensor Network for Water Monitoring. International Journal of Interactive Mobile Technologies (iJIM), [S.1.], v. 14, n. 11, p. pp. 288-296, jul. 2020. ISSN 1865-7923. https://doi.org/10.3991/ijim.v14i11.13681

[4] L. D. Xu, W. He and S. Li, "Internet of Things in Industries: A Survey," in IEEE Transactions on Industrial Informatics, vol. 10, no. 4, pp. 2233-2243, Nov. 2014.https://doi.org/10.1109/tii.2014.2300753

[5] "Internet of Things: Wireless Sensor Networks", White paper (International Electrotechnical Commission), International Electrotechnical Commission, Geneva, Switzerland, 2014

[6] ROSAS, João et al. Approach to Adapt a Legacy Manufacturing System into the IoT Paradigm. International Journal of Interactive Mobile Technologies (iJIM), [S.1.], v. 11, n. 5, p. pp. 91-104, jul. 2017. ISSN 1865-7923. https://doi.org/10.3991/ijim.v11i5.7073

[7] de Carvalho Silva, J., Rodrigues, J. J. P. C., Alberti, A. M., Solic, P., \& Aquino, A. L. L. (2017). LoRaWAN - A low power WAN protocol for Internet of Things: A review and opportunities. 2017 2nd International Multidisciplinary Conference on Computer and Energy Science (SpliTech), 1-6. https://doi.org/10.1109/ATSIP.2017.8075570

[8] Polonelli, T.; Brunelli, D.; Marzocchi, A.; Benini, L. Slotted ALOHA on LoRaWANDesign, Analysis, and Deployment. Sensors 2019, 19, 838. https://doi.org/10.3390 /s19040838 
[9] J. Ortín, M. Cesana and A. Redondi, "Augmenting LoRaWAN Performance with Listen Before Talk," in IEEE Transactions on Wireless Communications, vol. 18, no. 6, pp. 31133128, June 2019, https://doi.org/10.1109/twc.2019.2910512

[10] L. Beltramelli, A. Mahmood, P. Osterberg and M. Gidlund, "LoRa beyond ALOHA: An Investigation of Alternative Random-Access Protocols," in IEEE Transactions on Industrial Informatics, https://doi.org/10.1109/tii.2020.2977046

[11] [D. Zorbas, K. Abdelfadeel, P. Kotzanikolaou, and D. Pesch, "TS-LoRa: Time-slotted LoRaWAN for the Industrial Internet of Things," Computer Communications, vol. 153, pp. 110, 2020. https://doi.org/10.1016/j.comcom.2020.01.056

[12] A. Laya, C. Kalalas, F. Vazquez-Gallego, L. Alonso and J. Alonso-Zarate, "Goodbye, ALOHA!," in IEEE Access, vol. 4, pp. 2029-2044, 2016, https://doi.org/10.1109/ access.2016.2557758

[13] A. Kurtoglu, J. Carletta and K. Lee, "Energy consumption in long-range linear wireless sensor networks using LoRaWan and ZigBee," 2017 IEEE 60th International Midwest Symposium on Circuits and Systems (MWSCAS), Boston, MA, 2017, pp. 1163-1167, https://doi.org/10.1109/mwscas.2017.8053135

[14] P. S. Cheong, J. Bergs, C. Hawinkel and J. Famaey, "Comparison of LoRaWAN classes and their power consumption," 2017 IEEE Symposium on Communications and Vehicular Technology (SCVT), Leuven, 2017, pp. 1-6, https://doi.org/10.1109/scvt.2017.8240313

[15] Bouguera, T., Diouris, J. F., Chaillout, J. J., Jaouadi, R., \& Andrieux, G. (2018). Energy consumption model for sensor nodes based on LoRa and LoRaWAN. Sensors (Switzerland), 18(7), 1-23. https://doi.org/10.3390/s18072104

[16] A. M. Yousuf, E. M. Rochester, B. Ousat and M. Ghaderi, "Throughput, Coverage and Scalability of LoRa LPWAN for Internet of Things," 2018 IEEE/ACM 26th International Symposium on Quality of Service (IWQoS), Banff, AB, Canada, 2018, pp. 1-10, doi:. https://doi.org/10.1109/iwqos.2018.8624157

[17] A. Carlsson, I. Kuzminykh, R. Franksson and A. Liljegren, "Measuring a LoRa network: Performance possibilities and limitations", Proc. 18th Int. Conf. NEWAN 11th Conf. ruSMART, pp. 116-128, Aug. 2018. https://doi.org/10.1007/978-3-030-01168-0_11

[18] TEKTYTE Log4 data logger Datasheet 2020. https://www.tektyte.com/documents/TektyteLog4USB-Datasheet.pdf

[19] T. Chen, H. Kim, and Y. Yang, "Energy efficiency metrics for green wireless communications," 2010 International Conference on Wireless Communications and Signal Processing (WCSP), pp.1-6, 21-23 Oct.2010. https://doi.org/10.1109/wcsp.2010.5633634

[20] Kim, S.; Lee, H.; Jeon, S. An Adaptive Spreading Factor Selection Scheme for a Single Channel LoRa Modem. Sensors 2020, 20, 1008. https://doi.org/10.3390/s20041008

[21] AR-REYOUCHI, El Miloud et al. The Total Network Capacity of Wireless Mesh Networks for IoT Applications. International Journal of Interactive Mobile Technologies (iJIM), [S.1.], v. 14 , n. 08 , p. pp. 61-75, may. 2020. ISSN 1865-7923. https://doi.org/10.3991/ ijim.v14i08.12697

\section{Authors}

Aizat Faiz Ramli is currently a senior lecturer at Electronics Technology section, Universiti Kuala Lumpur British Malaysian Institute. He was the Head of Section for Postgraduate Studies and Research \& innovation Coordinator from February 2018 till February 2020 and 2017 till February 2018, respectively. Aizat Faiz was awarded $\mathrm{PhD}$ from the University of York, United Kingdom in 2014 and a Master of Engineer- 
ing degree in Electronic Engineering (MEng) from University of Hull, United Kingdom. His area of expertise and current research interest includes cognitive radio, artificial intelligence, wireless sensor networks and Internet of Things (IOT). Email: aizatfaiz@unikl.edu.my

Muhammad Ikram Shabry graduated from Universiti Kuala Lumpur British Malaysian Institute in Bachelor of Electronic Engineering Technology.

Mohd Azlan Abu received the B.Eng (HONS) in Electrical- Electronics from the Universiti Teknologi Malaysia, Malaysia, in 2008, M.Eng in Electrical-Electronics \& Telecommunication, from Universiti Teknologi Malaysia, Malaysia in 2010 and $\mathrm{PhD}$ in Electronic Engineering, from Universiti Putra Malaysia (UPM), Selangor in 2018. In 2008, he joined Motorola Technology Malaysia, as Research and Development Engineer. Since December 2010, he has been working in Universiti Kuala Lumpur British Malaysian Institute, Selangor, Malaysia, where he is a Senior Lecturer and Programme Coordinator for Bachelor of Engineering Technology in Electronics from 2015 until 2017. From 2017 until 2019, he was appointed as Head of Electronic Technology Section, UniKL BMI. Currently, he is appointed as Head of Section, Quality Assurance Section. His current research interests include Artificial Intelligence, IoT, Data Analytics, FPGA, Communication systems and Machine Learning.

Hafiz Basarudin is a senior lecturer at Universiti Kuala Lumpur British Malaysian Institute. He was previously Head of Section for Postgraduate studies. Dr Hafiz graduated with a PhD (2012) and MEng (2008) in electronics engineering from University of Hull (UK) and was a former UniKL BMI student (HND program). His area of expertise including radio propagation, satellite and meteorology.

Article submitted 2020-11-13. Resubmitted 2021-01-31. Final acceptance 2021-05-28. Final version published as submitted by the authors. 